\title{
Endoscopic Airway Findings in Infants with Obstructive Sleep Apnea
}

Anuja Bandyopadhyay ${ }^{1^{*}}$, Heather Muston ${ }^{1}$, James E Slaven², Hasnaa E Jalou1, William A Engle ${ }^{3}$ and Ameet S DaftaryMS ${ }^{1}$

${ }^{1}$ Department of Pediatrics, Section of Pediatric Pulmonology, Allergy and Sleep Medicine, Indiana University School of Medicine, Indianapolis, Indiana, USA ${ }^{2}$ Department of Biostatistics, Indiana University School of Medicine, Indianapolis, Indiana, USA

${ }^{3}$ Department of Pediatrics, Section of Neonatology, Indiana University School of Medicine, Indianapolis, Indiana, USA

\begin{abstract}
Objectives: This study describes the un-sedated endoscopic airway findings in infants with obstructive sleep apnea confirmed by polysomnography (PSG).

Methods: A retrospective chart review was performed on infants who had undergone both a documented airway endoscopy and PSG from 2006 to 2015 at our center. Demographics, comorbidities, findings of airway endoscopy and findings on PSG were extracted from the electronic medical record. Regression analyses were performed to determine the relationship between $\mathrm{AHI}$, endoscopic airway findings and gestational age.

Results: We identified 39 patients with PSG in room air and confirmed obstructive sleep apnea (Apnea Hypopnea Index (AHI) $>1$ /hour) who had undergone unsedated airway endoscopy. The median gestational age at time of PSG was 40 weeks and for endoscopy was 40 weeks. Median AHI on PSG was 17.2/hour. Laryngomalacia [87.2\%], pharyngomalacia [33.3\%] and tracheomalacia [10.3\%] were the three most prevalent findings on endoscopy. Surgically correctable fixed airway obstruction was uncommon. Prevalence of pharyngomalacia decreased with increased gestational maturity $(p=0.05)$. As the postmenstrual age at PSG completion increased, there was a trend towards a decline of the AHI $(p=0.087)$. Twenty-two (56.4\%) patients had a follow up PSG performed. Using paired $t$ test, there was a significant decrease in $\mathrm{AHI}(\Delta 13.41$ : $-40.9,15.8,15.8)$ from $23.4 /$ hour $(1.3-62)$ to $10.0 /$ hour $(0-32)$, despite no interim surgical intervention.
\end{abstract}

Conclusions: Dynamic airway collapse, including laryngomalacia and pharyngomalacia, were the most common findings in obstructive sleep apnea during infancy. The decreased prevalence of pharyngomalcia and trend towards improvement of $\mathrm{AHI}$ with time suggests airway immaturity contributes to obstructive sleep apnea observed during infancy and improves with age.

Keywords: Pharynx; Laryngomalacia; Tracheomalacia; Leukomalacia- periventricular; Sleep apnea-obstructive; Polysomnography; Infant-newborn; Bronchoscopy; Airway obstruction

Abbreviations: $\mathrm{PSG}=$ Polysomnogram; $\mathrm{AHI}=$ Apnea Hypopnea Index; $\mathrm{CO}_{2}=$ Carbon-dioxide; $\mathrm{ETCO}_{2}=$ End Tidal Carbon-dioxide; $\mathrm{BPD}=$ Bronchopulmonary Dysplasia

\section{Introduction}

Polysomnography [PSG] is the current gold standard test for diagnosis of sleep disordered breathing in adults and children, including neonates and infants [1]. There is a reported increased prevalence of sleep disordered breathing in former preterm children [2]. However, there is limited data on the clinical utility of PSG in neonates and infants [1], as well as a lack of epidemiologic data on the anatomic causes of obstructive sleep apnea in neonates and infants [3]. This patient population has anatomic and physiologic characteristics that place them at increased risk for scored respiratory events during polysomnography using the current scoring criteria from the American Academy of Sleep Medicine [4]. Neonates and infants are physiologically prone to sleep apnea due to immaturity of the respiratory control system $[5,6]$, increased airway collapsibility due to high airway compliance [7-9], craniofacial factors that narrow the airway lumen [10] and low functional residual capacity [11].

Endoscopic evaluation of infants with sleep associated upper airway obstruction under light anesthesia was first described more than 27 years ago [12]. Since this initial report only a limited number of studies have been published involving neonates and infants with PSG proven obstructive sleep apnea and subsequent management $[13,14]$. Recently, children 1.3 years to 1.8 years of age with obstructive sleep apnea were shown to have obstruction at the adenoid and tonsillar level in $89 \%$ and $82 \%$ of children, respectively [14]. However, there is no such available data in the neonatal and infant age group. There is a critical need to identify endoscopic findings in PSG proven neonatal obstructive sleep apnea to formulate subsequent therapeutic options.

The aim of this study was to describe the airway anatomic findings from unsedated airway endoscopies and associated comorbidities in neonates and infants with PSG confirmed obstructive sleep apnea. We hypothesize that neonates and infants with PSG confirmed obstructive sleep apnea have a high prevalence of dynamic airway abnormality that improves with age.

\section{Methods}

\section{Patient selection}

A retrospective chart review was performed to identify neonates and infants who had undergone a flexible airway endoscopy and also had documented obstructive sleep apnea on PSG between 2006 and 2015 at Riley Hospital for Children in Indianapolis, Indiana. Our institutional PSG database was searched for an obstructive apnea hypopnea index (AHI) of greater than 1 per hour to identify potential

*Corresponding author: Anuja Bandyopadhyay, Department of Pediatrics Section of Pediatric Pulmonology, Allergy and Sleep Medicine, Riley Hospital for Children at Indiana University Health, 705 Riley Hospital Dr. ROC 4270, Indianapolis, IN 46202-5225, Indiana, USA, Tel: 3179448602; Fax: 317 944-5791; E-mail: anubandy@iupui.edu

Received March 01, 2018; Accepted March 07, 2018; Published March 14, 2018

Citation: Bandyopadhyay A, Muston H, Slaven JE, Jalou HE, Engle WA, et al. (2018) Endoscopic Airway Findings in Infants with Obstructive Sleep Apnea. J Pulm Respir Med 8: 448. doi: 10.4172/2161-105X.1000448

Copyright: () 2018 Bandyopadhyay A, et al. This is an open-access article distributed under the terms of the Creative Commons Attribution License, which permits unrestricted use, distribution, and reproduction in any medium, provided the original author and source are credited. 
subjects and endoscopic findings were extracted from the medical record. Obstructive sleep apnea was defined as an AHI of greater than 1 per hour. Only PSGs performed in room air were included in our study, as oxygen use may affect the number of scored events. Patients were excluded if they did not have both a documented PSG and airway endoscopy in the electronic medical record. Patients who had any central apnea were excluded from the study since the focus of our chart review were infants with obstructive sleep apnea. Patients with cyanotic heart disease were excluded from this investigation. The study was performed with permission of the Institutional Review Board.

Patient demographics, comorbidities, birth gestational age, PSG findings and endoscopy findings were extracted from the electronic medical record. Postmenstrual age was defined as the sum of birth gestational age and chronological age. Prematurity was defined as birth gestational age less than or equal to 37 weeks. Comorbidities including diagnoses of bronchopulmonary dysplasia (BPD) (based on clinical assessment), oromotor incoordination (based on speech pathologist assessment), genetic abnormality (determined based on chromosomal microarray), craniofacial anomaly (based on clinical examination), intraventricular hemorrhage/periventricular leukomalacia/hydrocephalus (based on head ultrasound examination) and pulmonary hypertension (based on echocardiographic assessment) were obtained from neonatal discharge summaries.

\section{Polysomnography}

The PSGs were attended studies performed in the sleep laboratory and included the following monitoring parameters: electroencephalogram, electrooculogram, chin electromyogram, electrocardiogram, thermistor and pressure transducer airflow signals, respiratory inductance plethysmography or thoracic impedance measurement (prior to 2007), continuous oximetry and end tidal $\mathrm{CO}_{2}$ monitoring. The collection software was Sandman v 9.0 (Embla systems LLC, Tonawanda, NY). Oxyhemoglobin saturation was measured via pulse oximetry (Masimo,, Irvine, $\mathrm{CA}$ ). End tidal $\mathrm{CO}_{2}$ was measured at the nose using BCI Capnocheck (Smiths Medical PM, Inc Waukesha, WI) with side stream sampling. Nasal airflow was acquired with a nasal cannula (Salter Labs, Arvin, CA).

PSGs were staged per scoring guidelines from Anders, Parmalee and Emdee [15]. Before 2007, the institutional criteria followed for scoring respiratory events were: apnea for airflow limitation of $80 \%$ or greater from baseline for at least 2 breaths and hypopneas for airflow limitation of $30 \%$ or greater for at least 2 breaths duration associated with either $\geq 4 \%$ desaturation or an arousal. After 2007, AASM respiratory rules for children were followed for scoring respiratory events [16]. Before 2007, institutional criteria were followed for scoring arousals and awakenings. During NREM (quiet \& indeterminate) sleep, arousals were scored as change in EEG frequency from theta to alpha or beta or from delta to theta, alpha or beta for 3 seconds to 15 seconds. After 15 seconds the EEG change was scored as an awakening. All gross body movements were scored as either arousals or awakenings consistent with the duration of the event. In REM (active) sleep, in addition to an EEG frequency change, an additional elevation of EMG amplitude during the event was also required. After 2007, arousal indices were scored as per AASM scoring criteria [16]. Apneas were obstructive if activity was detected in the respiratory inductance plethysmography channels during airflow limitation. PSGs were reviewed and reported by board certified pediatric sleep medicine physicians.

\section{Bronchoscopy}

Flexible airway endoscopy was performed by a board certified pediatric pulmonologist at bedside following standard guidelines [17] The procedures were performed without sedation, using a Pentax FB$8 \mathrm{~V}$ bronchoscope with video processor and topical lidocaine.

\section{Statistical Analysis}

Results were analyzed for distributional fit, with outcomes having skewed distributions. Hence, results were reported as median (range) for continuous variables and frequency (percentage) for categorical variables. Regression analysis was performed to find a relationship between prevalence of various bronchoscopic findings and birth gestational age. Logistic regression analyses were performed to find a relationship between various endoscopic findings and comorbidities. Regression analyses were also performed to determine the presence of a relationship between $\mathrm{AHI}$ and birth gestational age, comorbidities, postmenstrual age at the time of sleep study and airway endoscopic findings. A p-value of 0.05 or below was considered statistically significant.

We collected data on follow up polysomnogram performed on patients who did not have any interim surgical intervention. We performed a paired $t$ test in order to determine if the change in $\mathrm{AHI}$ was significantly lower than zero. Analyses were performed with SAS v9.4 (SAS Institute, Cary, NC).

\section{Results}

Thirty-nine patients met our inclusion criteria. The demographics of the patient sample are described in Table 1. The indication for the PSG was clinical suspicion of upper airway obstruction. The median postmenstrual age at the time of PSG and endoscopy was close to 40 weeks. Oromotor incoordination and prematurity were the most common comorbidities. Of note, $15.4 \%$ of patients had neurologic sequelae such as Grade III or IV intraventricular hemorrhage (IVH), periventricular leukomalacia (PVL), and post hemorrhagic hydrocephalus (PHH). Twenty-nine (74.4\%) had a PSG and endoscopy performed within 7 days of one another.

The most common findings on airway endoscopy involved dynamic airway collapse, including laryngomalacia $(87.2 \%)$, pharyngomalacia (33.3\%) and tracheomalacia (10.3\%) (Table 2). Surgically treatable fixed obstructions were rare.

There was a positive association between periventricular leukomalacia and pharyngomalacia (Odds Ratio 15.63, 1.58-154.28, $\mathrm{p}=0.02$ ). There was no statistically significant relationship identified

\begin{tabular}{|l|l|}
\hline Demographics & $\mathrm{n}=39$ \\
\hline Sex (Male) & $18(46.2 \%)^{*}$ \\
\hline Birth Gestational age (weeks) & $36(26-41)^{+}$ \\
\hline Chronological age at the time of bronchoscopy (weeks) & $3.57(0.57-45.29)^{+}$ \\
\hline Post menstrual age at the time of bronchoscopy (weeks) & $40(36-71)^{+}$ \\
\hline Post menstrual age at the time of sleep study (weeks) & $40(36-52)^{+}$ \\
\hline Time interval between bronchoscopy and sleep study (days) & $4(0-142)^{+}$ \\
\hline Comorbidities & \\
\hline Oromotor incoordination & $27(69.2 \%)^{*}$ \\
\hline Prematurity & $21(53.9 \%)^{*}$ \\
\hline Bronchopulmonary dysplasia & $5(12.8 \%)^{*}$ \\
\hline Genetic abnormality & $4(10.3 \%)^{*}$ \\
\hline Periventricular leukomalacia & $6(15.4 \%)^{*}$ \\
\hline Craniofacial anomaly & $1(2.6 \%)^{*}$ \\
\hline Pulmonary Hypertension & $0(0 \%)^{*}$ \\
\hline Neuromuscular disorder & $0(0 \%)^{*}$ \\
\hline * Frequency (percentage); + Median (min-max) & \\
\hline
\end{tabular}

Table 1: Baseline characteristics. 


\begin{tabular}{|l|l|}
\hline \multicolumn{1}{|c|}{ Bronchoscopy Findings $(\mathbf{n = 3 9 )}$} & \multicolumn{1}{|c|}{$\begin{array}{c}\text { Frequency } \\
\text { (Percentage) }\end{array}$} \\
\hline Laryngomalacia & $34(87.2 \%)$ \\
\hline Pharyngomalacia & $13(33.3 \%)$ \\
\hline Tracheomalacia & $4(10.3 \%)$ \\
\hline Bronchomalacia & $0(0 \%)$ \\
\hline Abnormal Vocal Cord Motility & $0(0 \%)$ \\
\hline Subglottic Stenosis & $0(0 \%)$ \\
\hline Extrinsic Compression of Trachea & $1(2.6 \%)$ \\
\hline Choanal Atresia & $0(0 \%)$ \\
\hline Adenoidal Hypertrophy & $1(2.6 \%)$ \\
\hline Subglottic Cyst & $0(0 \%)$ \\
\hline
\end{tabular}

Table 2: Bronchoscopic findings.

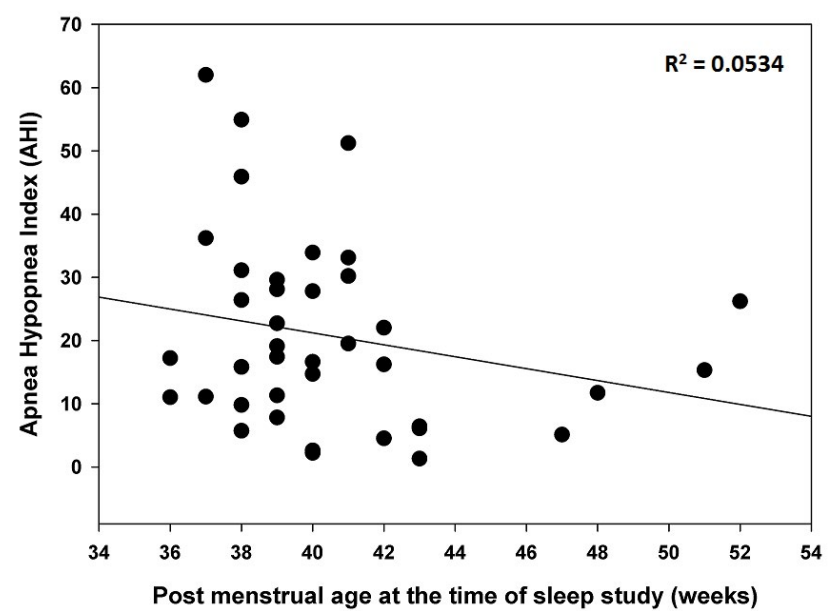

Figure 1: Relationship between post menstrual age and $\mathrm{AHI}(p=0.087)$.

\begin{tabular}{|l|l|}
\hline \multicolumn{1}{|c|}{ PSG findings } & \multicolumn{1}{|c|}{ Median (min-max) } \\
\hline AHI per hour & $17.2(1.3-62)$ \\
\hline Arousal Index per hour & $15.7(2.2-33.9)$ \\
\hline Mean ETCO21 $\mathrm{mmHg}^{3}$ & $40(30-52)$ \\
\hline Max ETCO21 $\mathrm{mmHg}^{3}$ & $54(40-80)$ \\
\hline Nadir SpO22 & $85(47-98)$ \\
\hline Mean SpO22 & $98.55(77-100)$ \\
\hline Percentage of sleep time with ETCO21 >45 $\mathrm{mmHg}^{3}$ & $1.9(0.1-20.1)$ \\
\hline Percentage of sleep time with SpO2 <90\% & $0.95(0-14.2)$ \\
\hline $\begin{array}{l}\text { ETCO }=\text { End-Tidal Carbon Dioxide Level; } \mathrm{SpO}_{2}=\text { Oxygen Saturation; } \\
\text { mmHg=Millimeters of Mercury }\end{array}$ \\
\hline
\end{tabular}

Table 3: PSG findings.

between laryngomalacia, tracheomalacia or bronchomalacia and any of the comorbidities.

The median AHI for our patients was 17.2/hour (1.3-62), the median mean-end-tidal $\mathrm{CO}_{2}$ was $40 \mathrm{~mm}$ of $\mathrm{Hg}$ (30-52) and median arousal index was $15.7 /$ hour (2.2-33.9). Other sleep parameters have been reported in Table 3 .

There was a trend towards a negative association between the AHI and postmenstrual age at the time of PSG (correlation coefficient $=-0.23$, $\mathrm{p}=0.087$ ). Figure 1 shows the relationship between postmenstrual age at time of PSG and AHI (Figure 2). Twenty-two (57\%) patients had a follow up PSG performed without interim surgical intervention. Using paired t-test, there was a significant decrease in AHI $(\Delta 13.41:-40.9$, $15.8,15.8$ ) from 23.4 /hour (1.3-62) to 10.0 /hour (0-32), despite no interim surgical intervention as shown in Figure 3.

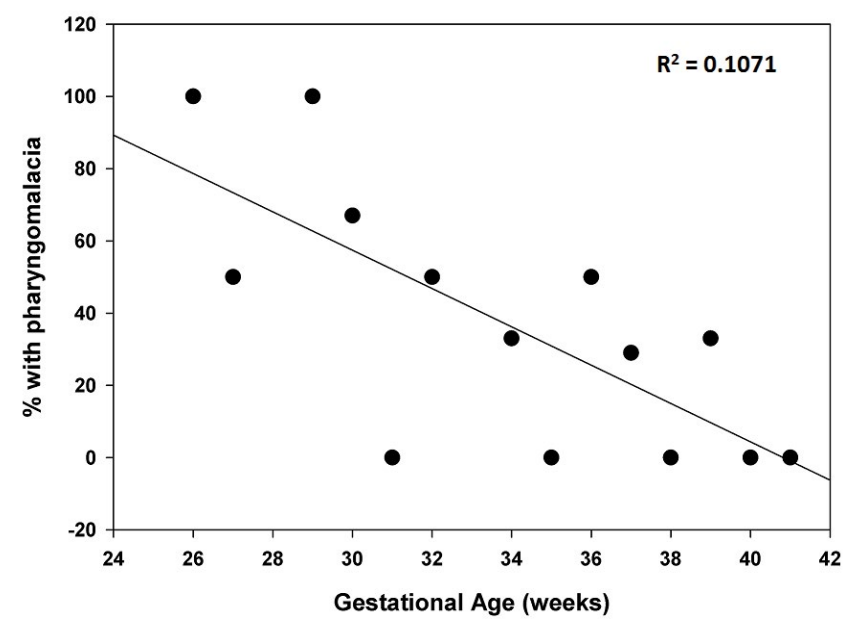

Figure 2: Prevalence of pharyngomalacia at various birth gestational ages $(p=0.04)$.

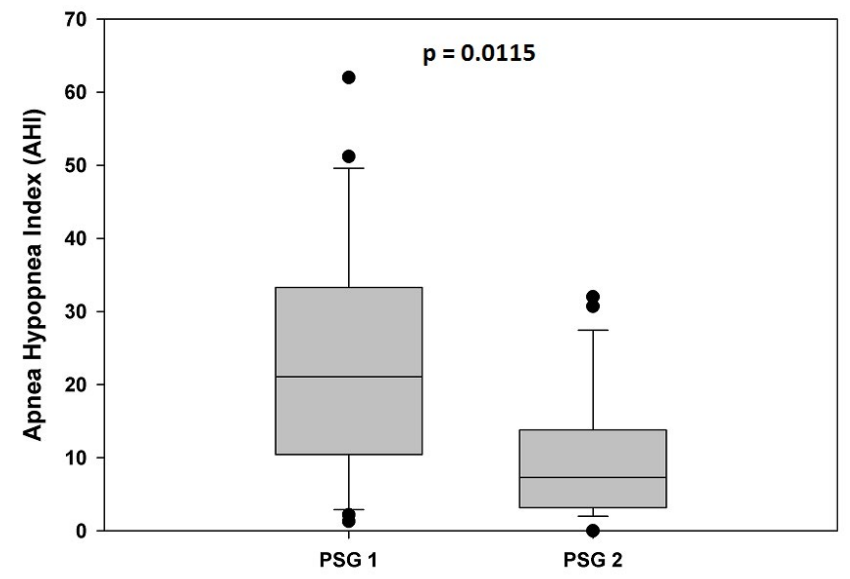

Figure 3: Change in $\mathrm{AHI}$ over time in 22 patients without interim surgical intervention.

\section{Discussion}

We have described the unsedated airway endoscopy findings in neonates and infants with PSG-confirmed obstructive sleep apnea. The findings suggest that dynamic airway collapse is more prevalent in obstructive sleep apnea during infancy than surgically correctable fixed airway obstruction. We also found that the prevalence of pharyngomalacia decreased with gestational maturation. Furthermore, there was improvement in the severity of sleep apnea over time without surgical intervention. These observations suggest that respiratory immaturity may be an important contributory factor to obstructive sleep apnea in neonates and infants.

$53.9 \%$ of our patients were premature, which validates existing literature showing a higher prevalence of sleep disordered breathing in the preterm population. However, only five (12.8\%) patients had significant underlying lung pathology such as bronchopulmonary dysplasia (BPD). Presence of underlying lung disease, such as BPD, results in decreased lung reserve and may affect the scoring of respiratory events during PSG. In the absence of significant lung pathology, the etiology of obstructive sleep apnea may be attributed 
to upper airway obstruction. Our study suggests that PSG confirmed obstructive sleep apnea in a neonates and infants is often associated with dynamic airway collapse.

Progressive resolution of airway obstruction caused by airway collapsibility has previously been reported in 2 moths to 12 month old infants [18]. Isono et al. [19] demonstrated that velopharyngeal closing pressure progressively decreased with increasing age. The proposed mechanisms include increased stiffness of soft tissue surrounding the upper airway [19], increased longitudinal tension of pharyngeal wall due to downward displacement of the larynx and hyoid bone as well as increased tracheal traction due to expansion in lung volume with age [20,21]. This explanation may justify, at least in part, the decreasing prevalence of pharyngomalacia and obstructive sleep apnea with advancing gestational maturation demonstrated in our study.

Our study is the first to report endoscopic findings in association with PSGs in this age group. Drug-induced sleep endoscopy (DISE), which is now standard of care, was not being routinely performed at our institution during the years from which our patient sample was obtained. Future studies, using drug-induced sleep endoscopy are needed to support the results demonstrated in this study in infants during their first year of life.

There was a trend towards a decrease in obstructive AHI with an increase in postmenstrual age at the time of PSG. This failed to achieve statistical significance, possibly due to the small sample size. We analyzed longitudinal data in a smaller subset of patients with follow up PSG, without any interim surgical intervention and found a statistically significant decrease in AHI for the paired samples. This observation from our data suggests that neonatal obstructive sleep apnea improves with time. This trend can be explained by physiologic airway maturation of neonates and infants.

Given the unique physiologic characteristics of neonates and infant and their predisposition to scored respiratory events under current pediatric PSG scoring guidelines, an AHI threshold for significant sleep apnea of one or more events per hour may be too liberal and result in over-diagnosis of neonatal obstructive sleep apnea. According to current consensus, pediatric sleep apnea is defined as severe for an AHI greater than 10 events per hour [22]. It may be worth reconsidering an alternative severity classification for sleep apnea in neonates and infants. There is an urgent need for normative PSG data in neonates to appropriately use this increasingly accessible diagnostic tool in clinical decision making, $15.4 \%$ of our patients had periventricular leukomalacia. There was a significant association between periventricular leukomalacia and pharyngomalacia (Odds Ratio 15.63, 1.58-154.28, $\mathrm{p}=0.02$ ). Due to the retrospective nature of the study, further data on the severity of periventricular leukomalacia were not consistently available. Periventricular leukomalacia is a form of white matter brain injury characterized by necrosis of white matter near lateral ventricles with diffuse gliosis in the surrounding white matter [23]. It is a strong predictor of motor disability such as cerebral palsy [24] and can also impact cognitive outcomes [25]. Our study is the first to report an association between pharyngeal dynamic collapsibility and periventricular leukomalacia. This association may be due to accompanying injury to centers in the brain responsible for muscle tone with secondary impact on pharyngeal muscles. Further studies reviewing detailed brain imaging of patients with periventricular leukomalacia and pharyngomalacia may better elucidate this association.

The primary weaknesses of our study are its retrospective and predominantly cross-sectional nature as well as use of unsedated flexible endoscopy for airway evaluation. Discharge diagnoses were obtained from discharge summary notes. Criteria establishing the diagnosis of bronchopulmonary dysplasia were not consistently documented. There was also a selection bias in this study, as patients were primarily in the NICU at the time of evaluation. Another significant limitation of this study is how the airway endoscopies were performed. The clinical practice of the institution at the young age of the patients was the primary reason for unsedated airway endoscopy. Obtaining airway evaluations in this manner can lead to increased dynamic airway abnormalities observed given the varying degrees of agitation and coughing at the time of procedure. However, this trade off needs to considered against the risk that deep sedation can mask airway dynamic abnormalities and there is increasing literature regarding neurocognitive effects from anesthesia in children [26]. Furthermore, an additional limitation of our study was that the findings on endoscopy, including the presence of airway malacia, was based on the subjective assessment of the endoscopist. There may have been inter-observer variability based on the experience and biases of the endoscopist. Unfortunately, due to unavailability of endoscopy videos, a review of those could not be performed by the authors. We therefore did not include severity assessment of the endoscopic findings due to this subjectivity. A prospective study with consistent grading of degree of airway obstruction will be useful in confirming our findings. Future studies, utilizing drug-induced sleep endoscopy are recommended to validate the observations in our study. Finally, this is the experience of a single center and practices may vary between institutions. We acknowledge a practice bias at our center, given access to a large pediatric sleep laboratory and capability to perform inpatient neonatal PSG.

\section{Conclusions}

Airway dynamic abnormalities are more common than surgically treatable fixed airway obstruction in obstructive sleep apnea in neonates and infants. This may be more a reflection of airway immaturity rather than true pathology in a significant number of neonates and infants. Prevalence of airway immaturity, particularly pharyngomalacia, increased with prematurity. AHI, the most common parameter to determine severity of obstructive sleep apnea, improved with time without any surgical intervention. Future multicenter prospective longitudinal studies are needed to describe the epidemiology of airway anatomical findings in neonatal obstructive sleep apnea, using standard bronchoscopy and sedation protocols.

\section{References}

1. DeHaan KL, Seton C, Fitzgerald DA, Waters KA, MacLean JE (2015) Polysomnography for the diagnosis of sleep disordered breathing in children under 2 years of age. Pediatr Pulmonol., 50: 1346-1353.

2. Rosen CL, Larkin EK, Kirchner HL, Emancipator JL, Bivins SF, et al. (2003) Prevalence and risk factors for sleep-disordered breathing in 8- to 11-year-old children: association with race and prematurity. J Pediatr 142: 383-389.

3. Katz ES, Mitchell RB, D'Ambrosio CM (2012) Obstructive sleep apnea in infants. Am J Respir Crit Care Med 185: 805-816.

4. Sanchez I, Vega-Briceno L, Munoz C, Mobarec S, Brockman P, et al. (2006) Polysomnographic findings in 320 infants evaluated for apneic events. Pediatr Pulmonol 41: 215-221.

5. Abu-Shaweesh JM, Martin RJ (2008) Neonatal apnea: What's new? Pediatr Pulmonol 43: 937-944.

6. Horemuzova E, Katz-Salamon M, Milerad J (2000) Breathing patterns, oxygen and carbon dioxide levels in sleeping healthy infants during the first nine months after birth. Acta paediatr 89: 1284-1289.

7. Gaultier C, Praud JP, Canet E, Delaperche MF, D'Allest AM (1987) Paradoxical 
Citation: Bandyopadhyay A, Muston H, Slaven JE, Jalou HE, Engle WA, et al. (2018) Endoscopic Airway Findings in Infants with Obstructive Sleep Apnea. J Pulm Respir Med 8: 448. doi: 10.4172/2161-105X.1000448

Page 5 of 5

inward rib cage motion during rapid eye movement sleep in infants and young children. J Dev Physiol 9: 391-397.

8. Heldt GP. (1988) Development of stability of the respiratory system in preterm infants. J Appl Physiol 65: 441-444.

9. Papastamelos C, Panitch HB, England SE, Allen JL (1985) Developmental changes in chest wall compliance in infancy and early childhood. J Appl Physiol 78: $179-184$

10. Arens R, Marcus CL (2004) Pathophysiology of upper airway obstruction: A developmental perspective. Sleep 27: 997-1019.

11. Kendig EL, Wilmott RW, Boat TF, Bush A, Chernick V (2012) Kendig and Chernick's disorders of the respiratory tract in children: Elsevier Health Sciences, USA.

12. Croft CB, Thomson HG, Samuels MP, Southall DP (1990) Endoscopic evaluation and treatment of sleep-associated upper airway obstruction in infants and young children. Clin Otolaryngol Allied Sci 15: 209-216.

13. Robison JG, Wilson C, Otteson TD, Chakravorty SS, Mehta DK (2013) Analysis of outcomes in treatment of obstructive sleep apnea in infants. Laryngoscope 123: $2306-2314$

14. Boudewyns A, Van de Heyning P, Verhulst S (2017) Drug-induced sedation endoscopy in children <2 years with obstructive sleep apnea syndrome: Upper airway findings and treatment outcomes. Eur Arch Otorhinolaryngol 274: 23192325.

15. Anders TF, Emde RN, Parmelee AH (1971) A manual of standardized terminology, techniques and criteria for scoring of states of sleep and wakefulness in newborn infants: UCLA Brain Information Service/BRI Publications Office, NINDS Neurological Information Network.

16. Medicine AAOS (2017) The AASM manual for the scoring of sleep and associated events: Rules. Terminology and Technical Specifications Westchester: AASM.

17. Green CG, Eisenberg J, Leong A, Nathanson I, Schnapf BM, et al. (1992) Flexible endoscopy of the pediatric airway. American Rev Respir Dis 145: 233235.

18. Isono S, Tanaka A, Ishikawa T, Nishino T (2000) Developmental changes in collapsibility of the passive pharynx during infancy. Am J Respir Crit Care Med 162: 832-836.

19. Bosma JF (1988) Functional anatomy of the upper airway during development Respiratory function of the upper airway. pp: 47-86.

20. Van de Graaff WB. (1988) Thoracic influence on upper airway patency. J Appl Physiol 65: 2124-2131.

21. Thut DC, Schwartz AR, Roach D, Wise RA, Permutt S, et al. (1993) Trachea and neck position influence upper airway airflow dynamics by altering airway length. J Appl Physiol 75: 2084-2090.

22. Mindell JA, Owens JA (2015) A clinical guide to pediatric sleep: Diagnosis and management of sleep problems: Lippincott Williams \& Wilkins, USA.

23. Kinney HC (2009) The encephalopathy of prematurity: One pediatric neuropathologist's perspective. Semin Pediatr Neurol 16: 179-190.

24. Skiöld B, Vollmer B, Böhm B, Hallberg B, Horsch S, et al. (2012) Neonatal magnetic resonance imaging and outcome at age 30 months in extremely preterm infants. J Pediatr 160: 559-66.

25. Woodward LJ, Clark CA, Bora S, Inder TE (2012) Neonatal white matter abnormalities an important predictor of neurocognitive outcome for very preterm children. PloS one 7: e51879.

26. Glatz P, Sandin RH, Pedersen NL, Bonamy AK, Eriksson LI, et al. (2017) Association of anesthesia and surgery during childhood with long-term academic performance. JAMA Pediatr 171: e163470. 